\title{
Correlation of Cognitive Performance and Thyroid Hormone Levels in Adolescents with Subclinical Hypothyroidism
}

\author{
A. R. Somashekar'1, Vishnu Girish ${ }^{2}$, Chandrika Rao ${ }^{1}$, Nandigudi Srinivas Murthy ${ }^{3}$ \\ ${ }^{1}$ Department of Pediatrics, M.S.Ramaiah Medical College and Hospital, Bangalore, India \\ ${ }^{2}$ M.S.Ramaiah Medical College and Hospital, Bangalore, India \\ ${ }^{3}$ Department of Patents and Research, M.S.Ramaiah Medical College and Hospital, Gokula Education \\ Foundation, Bangalore, India \\ Email:s arshekar2002@yahoo.com, vishnugirish@gmail.com, ${ }^{*}$ chandrikadoc@gmail.com, \\ nsmurthymsrmc@gmail.com
}

Received 21 March 2014; revised 20 April 2014; accepted 27 April 2014

Copyright (C) 2014 by authors and Scientific Research Publishing Inc.

This work is licensed under the Creative Commons Attribution International License (CC BY).

http://creativecommons.org/licenses/by/4.0/

(c) (i) Open Access

\begin{abstract}
Subclinical hypothyroidism (SCH) can negatively affect cognitive functioning. This study aimed at correlating serum T3, T4, TSH with adolescent's performance on a learning disability scale. Methods: A cross-sectional study was conducted on 100 schoolchildren, (10 - 15 years). Thyroid hormones were estimated and classified into two groups: euthyroid and subclinical hypothyroid. NIMHANS index for Specific Learning Disabilities was used to assess the learning ability and cognitive functions. Results: Subclinical hypothyroid group made more mistakes than euthyroid group. In SCH male group, T3 correlated with language and T4 levels correlated in all areas except in language. In the females, there is no significant correlation between $\mathrm{T} 3$ and ability parameters except in partial correlation coeffeicient among euthyroid children in arithmetic, visual-motor skills and memory. T4 results did not correlate in language skills. There was a statistical significance between T4 and ability skills in girls except in language. TSH and language skills correlated in females. Conclusion: T3 and T4 levels have correlation with cognitive skills other than TSH. It is necessary to measure both T3 and T4 in addition to TSH in adolescents.
\end{abstract}

\section{Keywords}

Subclinical Hypothyroidism, Adolescent Cognitive Performance, Thyroid Hormones

\footnotetext{
${ }^{*}$ Corresponding author.
}

How to cite this paper: Somashekar, A.R., et al. (2014) Correlation of Cognitive Performance and Thyroid Hormone Levels in Adolescents with Subclinical Hypothyroidism. Open Journal of Pediatrics, 4, 169-175. 


\section{Introduction}

Subclinical thyroid disease is characterized abnormal thyroid stimulating hormone (TSH) levels in presence of normal total of free thyroxine (T4) or triiodothyronine (T3) [1]. Its clinical significance has been widely debated and frequently encountered. There is no presently accepted protocol present for treatment of these patients. Studies [2]-[4], show that subclinical hypothyroidism (SCH) can negatively affect cognitive functioning. Academic performance being the prime focus of adolescents, it is important to evaluate cognitive functioning in SCH adolescents. Few studies exist on the correlation of thyroid hormone levels and cognitive function in SCH adolescents [5] [6]. This study aimed at correlating serum T3, T4, TSH with adolescent's performance on a learning disability scale.

\section{Methods}

A cross-sectional population based study was conducted on 100 schoolchildren of the age group 10 - 15 years studying in one school in Bangalore with similar socioeconomic status and financial background. Based on the (Mean $=10.32$, Standard Error $=0.16$ ) findings in a study carried out by $[6]$ Wu et al., with an alpha error of $5 \%$ and desired power of study at $80 \%$ the required sample size worked out to be 9 subjects in each group. It has also been reported that the prevalence of subclinical hypothyroidism in South India is around 9.4\% [7]. Thus in order to obtain the required number of children with subclinical hypothyroidism minimum of 100 children need to be screened. The above sample size was calculated to be sufficient to estimate differences in the language abilities of children also.

Children with history or suffering from Diabetics, epilepsy, anemia or with history of thyroidectomy, head injury, or on L-thyroxine therapy were excluded from study.

Socio-demographic data including age, gender, age of parents, occupation and education were collected. Informed consent was taken from the parents and also from the principals of the respective schools to collect the blood samples for estimating the thyroid hormone levels of the school children and administer the NIMHANS scale. Permission to conduct study was obtained from institutional ethics board.

Serum thyroxine $\left(\mathrm{T}_{4}\right)$, serum triiodothyronine $\left(\mathrm{T}_{3}\right)$ and thyroid stimulating hormone (TSH) was estimated in them using the RIA method. Based on the thyroid hormone levels the study population was classified into two groups: euthyroid and subclinical hypothyroid.

NIMHANS index for Specific Learning Disabilities (standardized scale—by NIMHANS) [8] was used to assess the learning ability and different aspects of cognitive functions of these children. The NIMHANS Index of SLD comprises test on language, arithmetic, visual-motor skills and memory. This data was collected by personal interview method. The number of mistakes made in each component of the index as well as the total number of mistakes made was recorded. This was used to correlate with their thyroid hormone levels. The number of mistakes in visual memory and auditory memory were added and was taken as one component-memory. Similarly the number of mistakes made in reading, spelling and comprehension was taken as the language component. The investigator administering NIMHANS scale was blinded to thyroid hormone levels.

We defined subclinical hypothyroidism as that grade of primary hypothyroidism in which there is an elevated thyroid-stimulating hormone (TSH) concentration more than $4.5 \mathrm{mIU} / \mathrm{L}$, in the presence of normal serum thyroxine (T4) and triiodothyronine (T3) concentrations and Subclinical Hyperthyroidism as TSH $<0.17 \mathrm{mIU} / \mathrm{L}$ [9].

\section{Statistical Analysis}

Data was analyzed using SPSS 18.0 (SPSS Inc., Chicago, USA.) A p value less than 0.05 was considered statistically significant. The descriptive statistics on SLD score were analyzed and expressed in median. Significance in differences in median values was done by Mann Whitney U test. The Spearman Rank correlation between the thyroid hormone levels (TSH, T3 and T4) in each group (euthyroid and subclinical hypothyroid) and the number of mistakes made in the NIMHANS index for SLD was calculated.

Statistical analysis of all the quantitative parameters such as scores relating to language, arithmetic, visualmotor skills and memory were summarized in terms of descriptive statistics such as mean, standard deviation. Student's t-test was employed to test for the difference in mean values between euthyroid and subclinical hypothyroid children. To express the relationship between the various thyroid hormone status such as T3, T4, TSH 
and different ability parameters (language, arithmetic, visual-motor skills and memory), both zero order correlation coefficient and partial correlation coefficient (controlled for age) were estimated.

\section{Results}

Out of the 100 school children, who formed the study population, 62 were girls and 38 were boys. There were 21 subclinical hypothyroid and 79 euthyroid children. There was no case of subclinical hyperthyroidism or any clinical manifestations of thyroid disorder.

Subclinical hypothyroid group made more mistakes when compared to the euthyroid group (Table 1).

The descriptive statistics for the various ability parameters (language, arithmetic, visual-motor skills and memory) for the children with euthyroid and subclinical hypothyroid children has been presented in Table 2. Children with subclinical hypothyroid status revealed higher mean score of 9.38 (language), 9.14 (arithmetic), 7.00 (visual-motor skills), 3.57 (memory) as compared to euthyroid children (8.67, 8.49, 6.28, 3.25 respectively.) in mistakes made in all the parameters. Similarly median values were also higher in subclinical hypothyroid group in Language, arithmetic and visual-motor skills as compared to euthyroid children. However none of the p values were significant.

The parameters were compared in the two thyroid status groups according to gender and $\mathrm{p}$ values were significant in females in arithmetic (Table 3).

Further attempt was made to control the effect of age along with gender and partial correlation coefficient was estimated (Table 4). The partial correlation coefficient values were found to be lower as compared to uncontrolled effect of age.

T3 levels in males correlated significantly with all the parameters in euthyroid group but only with language (0.036) in SCH.

T4 levels in males correlated significantly with all the parameters in euthyroid group (ranging from 0.83 0.98 ) and in SCH group (0.972 - 0.98) except in language in SCH, even after controlling for age. TSH and skill parameters in males did not correlate significantly in euthyroid and subclinical hypothyroid groups.

Table 1. Median of the number of mistakes made in each component of the NIMHANS index of SLD.

\begin{tabular}{ccc}
\hline Components & Subclinical Hypothyroid Median (Range) & Euthyroid Median (Range) \\
\hline Language & $8.00(6.00,11.50)$ & $7.00(5.00,12.00)$ \\
Arithmetic & $11.00(6.50,1200)$ & $9.00(6.00,12.00)$ \\
Visuo-Motor Skills & $8.00(4.00,11.00)$ & $6.00(4.00,8.00)$ \\
Memory & $3.00(2.00,5.00)$ & $3.00(2.00,4.00)$ \\
Total & $26.00(21.50,38.00)$ & $25.00(22.00,31.00)$ \\
\hline
\end{tabular}

Table 2. Descriptive statistics for language, arithmetic, visual-motor skills, memory according to thyroid status.

\begin{tabular}{ccccc}
\hline Status & & Language & Arithmetic & Visuo-Motor Skills \\
\hline Euthyroid N $=79$ & Median & 7.0 & 9.0 & 6.0 \\
Sub-Clinical Thyroid N $=\mathbf{2 1}$ & Median & $\mathbf{8 . 0}$ & $\mathbf{1 1 . 0}$ & $\mathbf{8 . 0}$ \\
\hline
\end{tabular}

\begin{tabular}{|c|c|c|c|c|c|c|}
\hline \multicolumn{7}{|c|}{ Test Statistics } \\
\hline & Age & Language & Arithmetic & Visuo-Motor Skills & Memory & Total \\
\hline $\mathrm{Z}$ & -1.121 & -1.225 & -1.139 & -0.957 & -0.831 & -1.194 \\
\hline Mean-p Value (2-Tailed) & 0.262 & 0.221 & 0.255 & 0.338 & 0.406 & 0.232 \\
\hline Median-p Value & 0.34 & 0.22 & 0.26 & 0.33 & 0.65 & 0.48 \\
\hline
\end{tabular}

a. Grouping Variable: thyroid status. 
Table 3. Descriptive statistics for the various parameters, by thyroid status and sex.

\begin{tabular}{|c|c|c|c|c|c|c|c|c|}
\hline Sex & & Thyroid Status & Language & Arithmtic & Visuo-Motor Skills & Memory & Total & Age \\
\hline \multirow{6}{*}{ Male } & & $N=29$ & & & & & & \\
\hline & Euthy & Mean & 8.72 & 8.59 & 6.52 & 3.21 & 32.69 & 12.07 \\
\hline & & Median & 7.00 & 9.00 & 6.00 & 3.00 & 29.00 & 12.00 \\
\hline & & $\mathrm{N}=9$ & & & & & & \\
\hline & Subcli & Mean & 7.78 & 7.33 & 5.22 & 2.56 & 28.56 & 11.56 \\
\hline & & Median & 8.00 & 8.00 & 5.00 & 2.00 & 24.00 & 11.00 \\
\hline \multirow{6}{*}{ Female } & & $N=50$ & & & & & & \\
\hline & Euthy & Mean & 8.64 & 8.44 & 6.14 & 3.28 & 32.28 & 12.18 \\
\hline & & Median & 7.50 & 9.00 & 6.00 & 3.00 & 31.00 & 13.00 \\
\hline & & $\mathrm{N}=12$ & & & & & & \\
\hline & Subclini & Mean & 10.58 & 10.50 & 8.33 & 4.33 & 40.50 & 11.92 \\
\hline & & Median & 9.00 & 12.00 & 8.00 & 4.00 & 38.00 & 12.50 \\
\hline Sex & & & Language & Arithmtic & Visuo-Motor Skills & Memory & Total & Age \\
\hline \multirow{2}{*}{ Males } & & $\mathrm{Z}$ & -0.656 & -0.507 & -0.727 & -0.614 & -0.533 & -0.937 \\
\hline & & Asymp. Sig. (2-tailed) & 0.512 & 0.612 & 0.467 & 0.540 & 0.594 & 0.349 \\
\hline \multirow{2}{*}{ Females } & & Z & -1.257 & -1.979 & -1.910 & -1.721 & -2.141 & -0.609 \\
\hline & & Asymp. Sig. (2-tailed) & 0.209 & 0.048 & 0.056 & 0.085 & 0.032 & 0.542 \\
\hline
\end{tabular}

In the females, in euthyroid and subclinical hypothyroid group, there was no significant correlation between T3 and ability parameters except in partial correlation coefficient among euthyroid children in arithmetic, visualmotor skills and memory.

There was statistically significant correlation between T4 and ability skills in girls of euthyroid and subclinical hypothyroid groups except in language.

Mean TSH in euthyroid group was 2.65 and in subclinical hypothyroid group was 5.58. There is no significant correlation between TSH and ability skills except in language after controlling for age in females.

\section{Discussion}

In our study the prevalence of subclinical hypothyroidism was $21 \%$. The prevalence of subclinical hypothyroidism in South India was found to be $9.4 \%$ in earlier study [7]. The population reference range of TSH is around 0.4 - $4.5 \mathrm{mIU} / \mathrm{L}$ and most patients with overt hypothyroidism have TSH above $10 \mathrm{mIU} / \mathrm{L}$. We have taken 4.5 $\mathrm{Miu} / \mathrm{l}$ as cut off value. However, several controversies surrounding the TSH reference range have surfaced in recent years.

We correlated serum T3, T4, TSH with adolescent`s performance on a learning disability scale. T3 and T4 showed significant correlation with the skills and TSH did not correlate with number of mistakes in both euthyroid and subclinical hypothyroid group. Children in subclinical hypothyroid group made more mistakes when compared to the children in the euthyroid group. Children with subclinical hypothyroid status revealed higher mean and median scores in mistakes made in all in all the four areas (language, arithmetic, visuospatial, memory) tested as compared to euthyroid children.

Earlier study by Wu et al. in American adolescents [6] showed that cognitive assessment scores on average tended to be lower in adolescents with subclinical hyperthyroidism and higher in those with subclinical hypothyroidism than the score for the euthyroid group. Almeida et al. [10] showed no difference in performance of the psychological tests between patients with subclinical hypothyroidism and without thyroid disease. Ayca et al. 
Table 4. Zero order correlation coefficients with T3, T4, and TSH with each component of NIMHANS Index for SLD according thyroid status and partial correlation coefficients adjusted for age ${ }^{*}$.

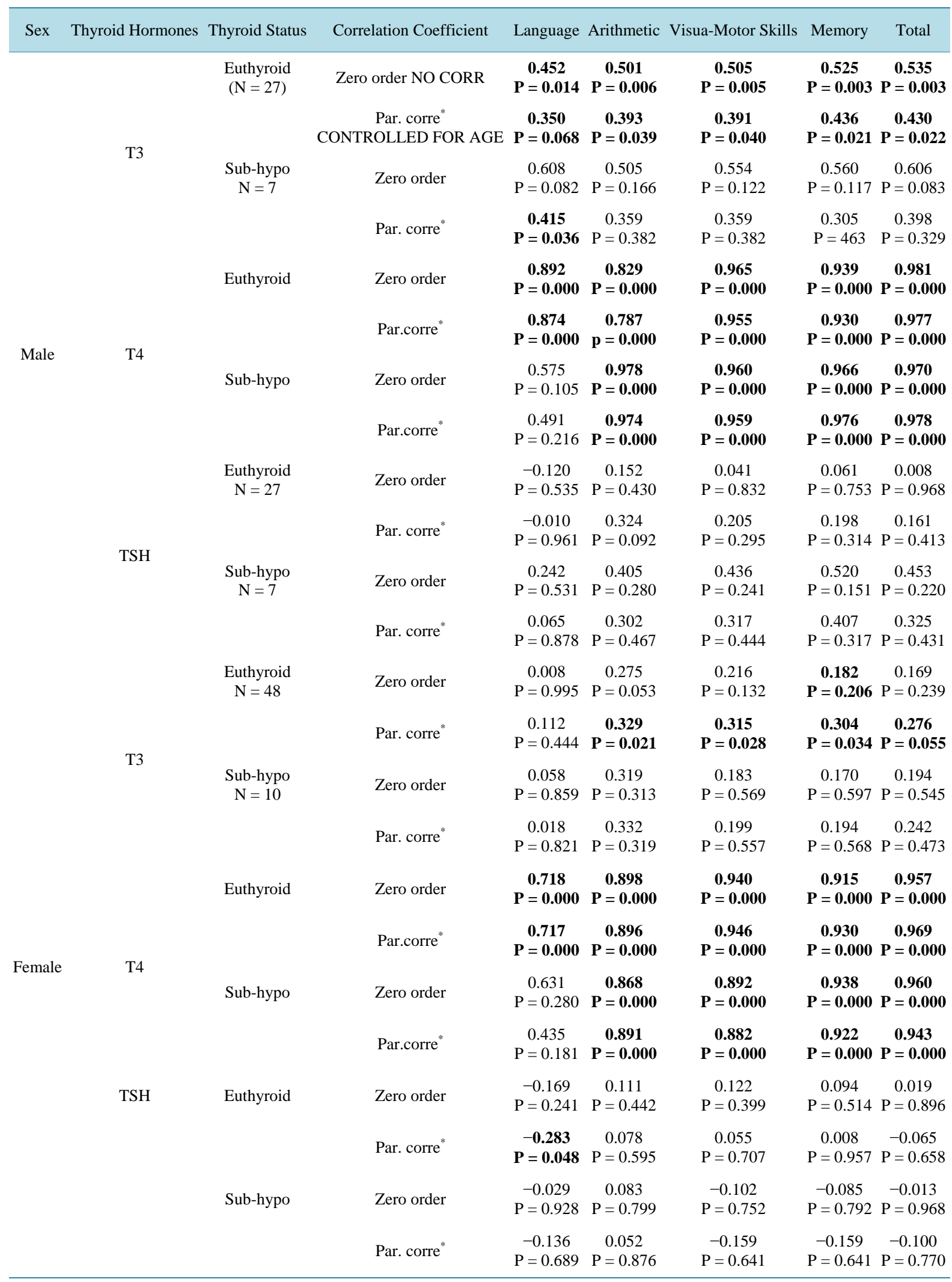


in their study [11] demonstrated that pediatric patients with SCH showed poor performance in tests measuring attention but no difference in intelligence. Similar study by Aijaz et al. [12] showed increase in attention deficit in SCH children but normal verbal and visual processing abilities.

Thyroid hormones are necessary for maturation of the brain and its functions throughout life and especially in certain windows of growth [13] [14] to enable timely migration of the cells. In the brain T3 is mostly derived from local T4 deiodination (D2 and D3).In thyroid deficiency, increased D2 activity maintains normal T3 concentration despite reduced T4 concentrations in brain. An important fact is that thyroid hormones are present in CSF at less than ten times the serum concentrations [15]. The findings of this study direct attention to the necessity to measure both T3 and T4 in addition to TSH in adolescents.

This study was the first of its kind done on adolescents in India. The high prevalence of subclinical hypothyroidism as found out by this study, as well as the fact that there are more adolescents in the high normal range of TSH reference range, calls out the need for a large study to find out the prevalence of these subclinical thyroid disorders in the Indian population

The limitation of our study is the relative small sample size of SCH children. The second limitation of this study was that only one blood sample value was taken and transient SCH or diurnal variation was not considered. We have not studied cognitive abilities after therapy in SCH children. There are contrary reports of treating SCH and the role of combined T3 and T4 administration in adolescents particularly needs to be studied [16].

\section{Conclusion}

Further, more interventional studies relating to subclinical hypothyroidism and cognitive functioning which can throw light on the mild changes in cognition which may not be picked up by the usual cognitive tests, need to be done. This is especially important in India as adolescents who are in the threshold of higher studies, decreased cognitive function may be a determent to achieve high standard in academics.

\section{References}

[1] Biondi, B. and Cooper, D.S. (2008) The Clinical Significance of Subclinical Thyroid Dysfunction. Endocrine Reviews, 29, 76-131. http://dx.doi.org/10.1210/er.2006-0043

[2] Correia, N., et al. (2009) Evidence for a Specific Defect in Hippocampal Memory in Overt and Subclinical Hypothyroidism. The Journal of Clinical Endocrinology \& Metabolism, 94, 3789-3797. http://dx.doi.org/10.1210/jc.2008-2702

[3] Ceresini, G., et al. (2009) Thyroid Function Abnormalities and Cognitive Impairment in the Elderly. Results of the InCHIANTI Study. Journal of the American Geriatrics Society, 57, 89-93. http://dx.doi.org/10.1111/j.1532-5415.2008.02080.x

[4] Baldini, I.M., et al. (1997) Psychopathological and Cognitive Features in Subclinical Hypothyroidism. uro-Psychopharmacology \& Biological Psychiatry, 21, 925-935. http://dx.doi.org/10.1016/S0278-5846(97)00089-4

[5] Samuels, M.H. (2010) Cognitive Function in Subclinical Hypothyroidism. The Journal of Clinical Endocrinology \& Metabolism, 95, 3611-3613. http://dx.doi.org/10.1210/jc.2010-1242

[6] Wu, T., Flowers, J.W., Tudiver, F., Wilson, J.L. and Punyasavatsut, N. (2006) Subclinical Thyroid Disorders and Cognitive Performance among Adolescents in the United States. BMC Pediatrics, 19, 6-12.

[7] Usha Menon, V., Sundaram, K.R., Unnikrishnan, A.G., Jayakumar, R.V., Nair, V. and Kumar, H. (2009) High Prevalence of Undetected Thyroid Disorders in an Iodine Sufficient Adult South Indian Population. Journal of the Indian Medical Association, 107, 72-77.

[8] Kapur, M., John, A., Rozario, J. and Oommen, A. (1991) NIMHANS Index for Specific Learning Disabilities. Department of Clinical Psychology, NIMHANS Publication, Bangalore.

[9] Almandoz, J.P. and Gharib, H. (2012) Hypothyroidism: Etiology, Diagnosis, and Management. Medical Clinics of North America, 96, 203-221. http://dx.doi.org/10.1016/j.mcna.2012.01.005

[10] Almeida, C., Vaisman, M., Costa, A.J.L., Reis, F.A.A., Reuters, V., Teixeira, P., Ferreira, M., Teixeira, L.B.B.M., Araujo, G.R.B. and Brasil, M.A. (2007) Are Neuropsychological Changes Relevant in Subclinical Hypothyroidism? Arquivos Brasileiros de Endocrinologia \& Metabologia, 51, 606-611. http://dx.doi.org/10.1590/S0004-27302007000400016

[11] Ayca, T.E., Yaseman, T., Evsen, A., Efnan, M., Emel, E., Bakar, T.S. (2012) Journal of Clinical Research in Pediatric Endocrinology, 4, 21-24. http://dx.doi.org/10.4274/Jcrpe.497

[12] Aijaz, N.J., Flaherty, M.E., Preston, T., Bracken, S.S., Lane, A.H. and Wilson, T.A. (2006) Neurocognitive Function in 
Children with Compensated Hypothyroidism: Lack of Short Term Effects on or off Thyroxine. BMC Endocrine Disorders, 6, 2. http://dx.doi.org/10.1186/1472-6823-6-2

[13] Bernal, J. (2007) Thyroid Hormones Receptors in Brain Development and Function. Nature Clinical Practice Endocrinology \& Metabolism, 3, 249-259. http://dx.doi.org/10.1038/ncpendmet0424

[14] Alvarez-Dolado, M., Ruiz, M., del Rio, J.A., et al. (1999) Thyroid Hormone Regulates Reelin and Dab 1 Expression during Brain Development. Journal of Neuroscience, 19, 6979-6973.

[15] Larsen, P.R., Silva, J.R. and Kaplan, M.M. (1981) Relationship between Circulating and Intracellular Thyroid Hormones. Physiological and Clinical Implications. Endocrine Reviews, 2, 87-102. http://dx.doi.org/10.1210/edrv-2-1-87

[16] Hennemann, G., Docter, R., Visser, T.J., Postema, P.T. and Krenning, E.P. (2004) Thyroxine Plus Low-Dose, SlowRelease Triiodothyronine Replacement in Hypothyroidism: Proof of Principle. Thyroid, 14, 271-275. http://dx.doi.org/10.1089/105072504323030924 